\title{
GMR
}

\section{Transplantation of erythropoietin gene- transfected umbilical cord mesenchymal stem cells as a treatment for limb ischemia in rats}

\author{
J.P. Li ${ }^{1}$, D.W. Wang ${ }^{2}$ and Q.H. Song ${ }^{3}$ \\ ${ }^{1}$ Peripheral Vascular, Henan Province Hospital of Traditional Chinese Medicine, \\ Zhengzhou, China \\ ${ }^{2}$ Peripheral Vascular, Shandong College of Traditional Chinese Medicine, \\ Laiyang, China \\ ${ }^{3}$ Peripheral Vascular, Tianjin People's Hospital, Tianjin, China \\ Corresponding author: D.W. Wang \\ E-mail: wangdaweimm@163.com \\ Genet. Mol. Res. 14 (4): 19005-19015 (2015) \\ Received August 13, 2015 \\ Accepted October 1, 2015 \\ Published December 29, 2015 \\ DOI http://dx.doi.org/10.4238/2015.December.29.8
}

\begin{abstract}
The aim of this study was to investigate the mechanisms of erythropoietin (EPO)-transfected umbilical cord mesenchymal stem cells (UC-MSCs) in the treatment of rats with ischemic limb and provide a theoretical basis for optimization of UC-MSC transplantation. Sixty SD rats were randomly divided into four groups: ischemia control group, EPO treatment group, UC-MSCs treatment group, EPO gene transfected UCMSC treatment groups. (15 rats in each group). The left femoral hind artery and its branches were ligated to develop hind limb ischemia in male SD rats. Five points were injected in the adductor and gastrocnemius muscles with medium, cDNA3-EPO gene DNA-liposome complex solution or UC-MSCs in control groups and EPO-transfected-UC-MSCs in the experimental group. Western blot confirmed in vitro EPO expression in EPO gene-transfected human UC-MSCs. Arterial angiography at 4 weeks post-transplantation showed no development of blood vessels in the control group and moderate angiogenesis in the EPO- and UC-MSC-treated groups. However, a large
\end{abstract}


number of freshmen angiogenesis and abundant collateral circulation was observed in the EPO-transfected-UC-MSC-treated experimental group. Rat capillary density measurement results confirmed the angiographs quantitatively and showed no statistically significant difference between EPO- and UC-MSC-treated groups $(P>0.05)$. CM-Dil-positive cell numbers were $(0 \pm 0.00),(0 \pm 0.00),(32.46 \pm 6.68),(59.64 \pm 10.38) / \mathrm{HP}(\mathrm{P}<0.05)$. RT-PCR detected that the in vivo mRNA expression of the EPO gene was relatively higher in the EPO-transfected-UC-MSC-treated group than the EPO-treated group $(0.79 \pm 0.06$ vs $0.19 \pm 0.04, \mathrm{P}<0.05)$. Thus, this study revealed that using UC-MSCs as vector in gene therapy for limb ischemia facilitates stable and effective expression of EPO compared to direct gene injection.

Key words: Mesenchymal stem cells; Transplantation; Ischemia; Rats; Erythropoietin

\section{INTRODUCTION}

Umbilical cord mesenchymal stem cells (UC-MSCs) are a group of cells that have multiple differentiation potency, characteristics of easy separation, high proliferative rate, and a stable genome. It is known for its potency to promote repair of various in vivo tissue lesions and ability to differentiate into multiple mesenchymal cells on in vitro proliferation (Kidd, 1995; Chi et al., 2006; Park et al., 2007). Upon induction under specific in vitro conditions, UC-MSCs has been shown to differentiate into bone, cartilage, fat, tendons and muscles, and other mesodermal cells (Li et al., 2010; Park et al., 2014; Cui et al., 2014). In recent years, studies on stem cell transplantation with subsequent directed differentiation of transplanted cells for gene therapy and those that aim to improve transplantation efficacy have been gaining attention (Bieback et al., 2004; Chen et al., 2005; Jin et al., 2007).

Erythropoietin (EPO) has recently been known to promote proliferation of human MSCs (Jin et al., 2011) as well as the deferment and differentiation of endothelial progenitor cells and guide the homing of vascular endothelial cells to form blood vessels (Marfia et al., 2011). In this study, we used pcDNA3-EPO plasmid carrying the erythropoietin gene to transfect human UCMSCs, which were then transplanted into ischemic lower limbs in rats in order to understand the therapeutic mechanisms and efficiency of this method. This would also provide a theoretical basis for optimizing UC-MSC transplantation in the treatment of limb ischemia.

\section{MATERIAL AND METHODS}

\section{Animals, reagents, and instruments}

Sprague Dawley (SD) rats weighing between 250 and $300 \mathrm{~g}$ were purchased from the Animal Laboratory (animal quality certification No. SCXK20070024), Chinese Academy of Medical Sciences. Of the 60 animals, half were male. During the experiments, animals were disposed according to the requirements of animal ethics. DMEM/F12 medium, 5\% fetal bovine serum (FBS), and 25\% Trypsin were purchased from Gibco BRL Corporation, USA. Trizol and Lipofectamine ${ }^{\circledR}$ 
2000 was from Invitrogen, USA. Streaming antibodies for CD31, CD34, CD45, CD73, CD105, HLA-DR, VWF, KDR, and CD235a were obtained from Beckman Coulter, Inc., USA and Sigma for sorting by flow cytometry. Other reagents included RT-PCR reverse transcription kit (Promega, Madison, WI, USA), Taq enzyme (Canada BBI), dNT (TaKaRa), western blot chemiluminescence reagent (Santa Cruz), rabbit anti-human EPO antibody, HRP-goat anti-rabbit IgG, PVDF membrane (Pierce), and $5 \mu \mathrm{L}$ micro-syringes (Hamilton, USA). Key laboratory equipment used in this study included carbon dioxide incubator (UK RS Biotech); Clean Benches (Suzhou Aetna air technology) and inverted fluorescence microscope (Japan Nikon Corporation).

\section{Preparation and identification of UC-MSCs}

This study was approved by the Ethics Committee of Henan Province Hospital. Umbilical cords from healthy full-term fetuses delivered through Cesarean section were obtained. After thorough rinsing with PBS, the interstitial tissue excluding the vessels was cut into tissue blocks the size of a diameter of about $1 \mathrm{~mm}^{3}$. Type II collagen enzymes 0.1 and $0.25 \%$ trypsin were used for digestion at $37^{\circ} \mathrm{C}$ for $30 \mathrm{~min}$. After centrifugation, the cells were seeded on culture dishes containing DMEM medium with $5 \% \mathrm{FBS}$. After the first passage, each passage lasted 3 days with an amplification ratio of 1:3. Passage 3 (P3) cells were digested and labeled with PE FITC-conjugated CD73, CD105, HLA-DR, CD31, VWF, KDR, CD34, CD45, CD235a streaming antibodies. Flow cytometry was used for detection.

\section{EPO gene transfection and detection in vitro}

UC-MSCs of P3 generation were transfected with pcDNA3-EPO plasmid containing the the EPO gene by liposome-mediated transfection method using Lipofectamine ${ }^{\circledR} 2000$, according to the manufacturer instructions. After $24 \mathrm{~h}$, cells were seeded on six-well plates (1:10) and incubated with fresh complete medium. Next day, the medium was replaced with enough screening medium containing $400 \mathrm{mg} / \mathrm{L}$ G418 to last for 6 days, after which medium containing $200 \mathrm{mg} / \mathrm{L} \mathrm{G} 418$ was used. Two weeks later, single clones were picked from the positive clones and passaged.

For detection of EPO expression in EPO-transfected UC-MSCs, total protein was extracted from $1 \times 10^{7}$ stably transfected UC-MSCs and cultured fetal liver cell line L02 (positive control). Upon collection, RIPA buffer (containing $10 \mathrm{~g} / \mathrm{L}$ PMSF) was added and incubated in ice bath. The solution was then centrifuged at $4^{\circ} \mathrm{C}$ and the supernatant collected. SDSpolyacrylamide gel electrophoresis was performed after determination of protein concentration. The proteins were transferred to a PVDF membrane, incubated in the blocking solution followed by rabbit anti-human EPO polyclonal antibody (1:200) and HRP-goat anti-rabbit IgG (1:5000) with intermittent washes. The blots were imaged at a 1-min exposure to chemiluminescence reagents using an image analyzer.

\section{CM-Dil labeling of UC-MSCs}

CM-Dil-labeling solution was prepared by adding $1 \mathrm{~mL}$ complete medium to $5 \mu \mathrm{L}$ CM-Dil liquid. This solution was added at $40 \mu \mathrm{L} / \mathrm{cm}^{2}$ to adherent cells (90\% confluence) after three PBS washes. After incubation in a humidified chamber at $37^{\circ} \mathrm{C}, 5 \% \mathrm{CO}_{2}$ for $20 \mathrm{~min}$, the labeling solution was replaced with $5 \mathrm{~mL}$ medium and incubated for 10 min under same conditions followed by 
two washes. The effect of CM-Dil labeling effect and morphological changes in the cultures were observed under inverted fluorescence microscope after $24 \mathrm{~h}$. Both total and CM-Dil-positive cells were counted in five horizons selected at random. The labeling efficiency was calculated as the number of label-positive cells in the visual field/total number of cells in the visual field $x 100 \%$. The cells were washed thrice with PBS before transplantation.

\section{Preparation of animals and bone marrow transplantation}

The SD rats were divided into four groups of 15 each and anesthetized with intraperitoneal injection of urethane at $1.2 \mathrm{~g} / \mathrm{kg}$. A longitudinal incision was made from the left groin to the knee ligament and the femoral artery and its branches were ligated. Starting at a distance of $2 \mathrm{~cm}$ below the ligation, five points with $0.2-\mathrm{cm}$ intervals were marked with micropipettes on the adductor and gastrocnemius. Through each point, $60 \mu \mathrm{L}$ solution was injected in all tested subjects. Rats in the ischemic control group and the EPO-treated group were injected with culture medium and pcDNA3-EPO DNA-liposome complexes containing $5 \mu \mathrm{g}$ gene, respectively. For UC-MSC- and EPO-transfected-UC-MSCs-treated groups, the rats were injected with $3 \times 10^{6}$ UC-MSCs and UCMSCs containing EPO gene, respectively. The subjects were observed for 5 min and layer wound closure was performed if nothing was abnormal.

\section{Angiography}

At 4 weeks post-surgery, the abdominal aorta of each experimental animal was punctured and a bolus injection of $2 \mathrm{~mL} 70 \%$ diatrizoate was administered. Continuous DSA radiography was performed while monitoring using X-ray.

\section{Determination of capillary density}

Animals were sacrificed and the left gastrocnemius muscle tissues were collected to prepare paraffin sections. Mouse anti-rat factor VIII antibody (1:50, NeoMarker Biotech, Beijing, China) and goat anti-mouse IgG antibody-HRP polymer (Beijing Zhongshanbio Technology) were used for immunohistochemistry staining. For each sample, 20 horizons were randomly selected; while the number of newly formed vessels was calculated at high magnification (400X), the number of CM-Dil-positive cells in each horizon was counted under fluorescence and the mean of each group was used for further analysis.

\section{RT-PCR analysis for EPO gene expression in vivo}

The transplanted areas in rats were powdered with liquid nitrogen. After pulsing and shaking with Trizol, the samples were centrifuged for $5 \mathrm{~min}$. Anhydrous ethanol was added into the supernatant, mixed, and the contents were transferred to UNQ-10 column for centrifugation. RNA samples in the tube were collected, and used immediately or preserved at $-70^{\circ} \mathrm{C}$. cDNA was synthesized using the Reverse Transcription system Kit (Promega) according to the manufacturer protocol. PCR primers were designed based on the full-length EPO cDNA sequences in GenBank and synthesized by the Shanghai Biological Engineering Company. We amplified upstream primer of EPO cDNA full-length sequence: 5'-ATGAATTCATGGGGGTGCACGAATGTCC-3', downstream primer: 
5'-GCCTCGAGTCATCTGTCCCCTGTCCTGC-3', and the expected amplified fragment length was $582 \mathrm{bp}$. In each group RT-PCR method was used to identify the upstream primer EPO expression: 5'-TCATCTGTGACAGCCGAGTC-3', downstream primer: 5'-CACTGACGGCTTTATCCACA-3', and the expected amplified fragment length was $288 \mathrm{bp}$. $\beta$-actin (reference gene) primers $5^{\prime}$-AGA GGGAAATCGTGCGTGAC-3' (upstream) and 5'-ACATCTGCTGGAAGGTGGAC-3' (downstream) were used to obtain a 224-bp product. PCR conditions were set as: 30 cycles were performed after initial denaturation at $96^{\circ} \mathrm{C}$ for 5 min followed by 30 cycles each of $95^{\circ} \mathrm{C}$ for $30 \mathrm{~s}, 60^{\circ} \mathrm{C}$ for $30 \mathrm{~s}$, and $72^{\circ} \mathrm{C}$ for $30 \mathrm{~s}$ with a final extension period at $72^{\circ} \mathrm{C}$ for $10 \mathrm{~min}$. Following electrophoresis on $2 \%$ agarose gel, the PCR products were imaged and the bands quantified using an image analysis system. The relative content of EPO mRNA was calculated based on the optical density of the PCR products of the internal reference ( $\beta$-actin).

\section{Statistical methods}

This multi-group experiment was based on a completely randomized design. All data analysis was performed using the SPSS 17.0 software. All measurements are reported as means \pm standard deviation. After variance homogeneity test, ANOVA was used for comparison. If the data was statistically significant, the least significant difference (LSD) method was used for pairwise comparison. Throughout, $\mathrm{P}<0.05$ was considered to be statistically significant.

\section{RESULTS}

\section{Morphology of UC-MSCs under cell culture conditions}

The adherent cell cultures showed significant proliferation in 4 or 5 days. Cells were fusiform or polygonal with the emergence of mitosis, whereas some cloned cells had colony-like growth. The final morphology was single, long, spindle-shaped, with the cell center enlarged. After 8-9 days, the cells covered the surface of the dish in swirl-shaped arrangements. While the center of the vortices had stratified growth, most of the cells were in a swirling or radial monolayer (Figure 1). Flow cytometry analysis showed that the cells were positive for CD29, CD49, CD90, CD105, and HLA-1, and the homogeneity of the UC-MSCs was high with $>96 \%$ purity.
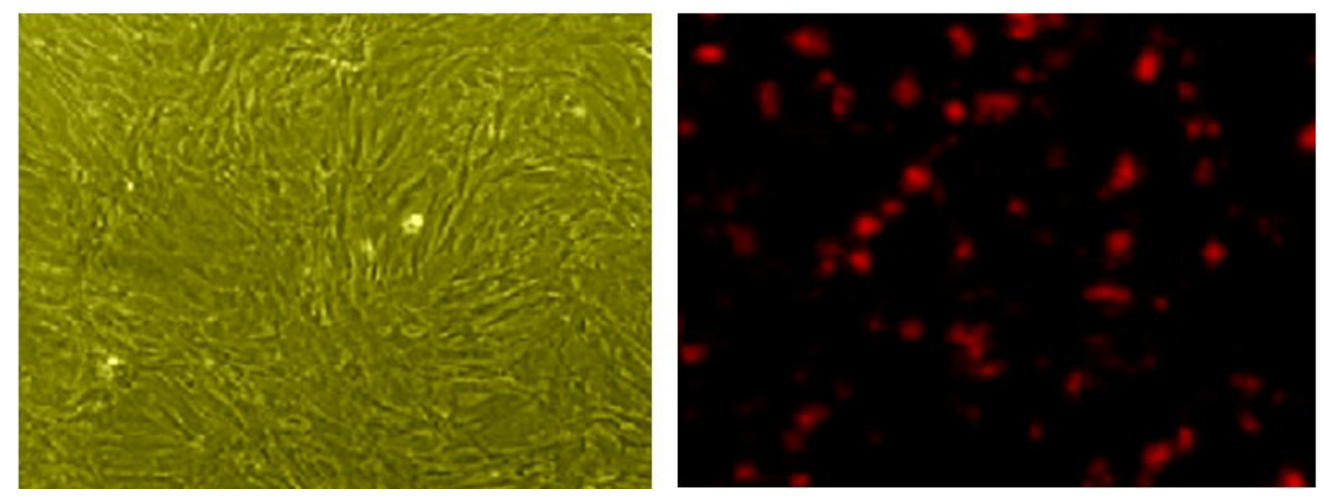

Figure 1. Morphology of umbilical cord mesenchymal stem cells in culture. 


\section{Validation of umbilical cord mesenchymal stem cell phenotype in vitro}

Cell type-specific markers in adherent UC-MSCS could be detected with $100 \%$ efficiency using flow cytometry. High expression of umbilical cord mesenchymal stem cell-specific markers namely CD29, CD49, CD90, CD105 and HLA-1 was detected in the cultured UC-MSCs. No or low expression of hematopoietic cell markers CD34 and CD45 as well as endothelial cell marker CD31 were observed (Figure 2). At 24 h, CM-Dil-labeled UC-MSCs could be visualized in red under a fluorescence microscope.
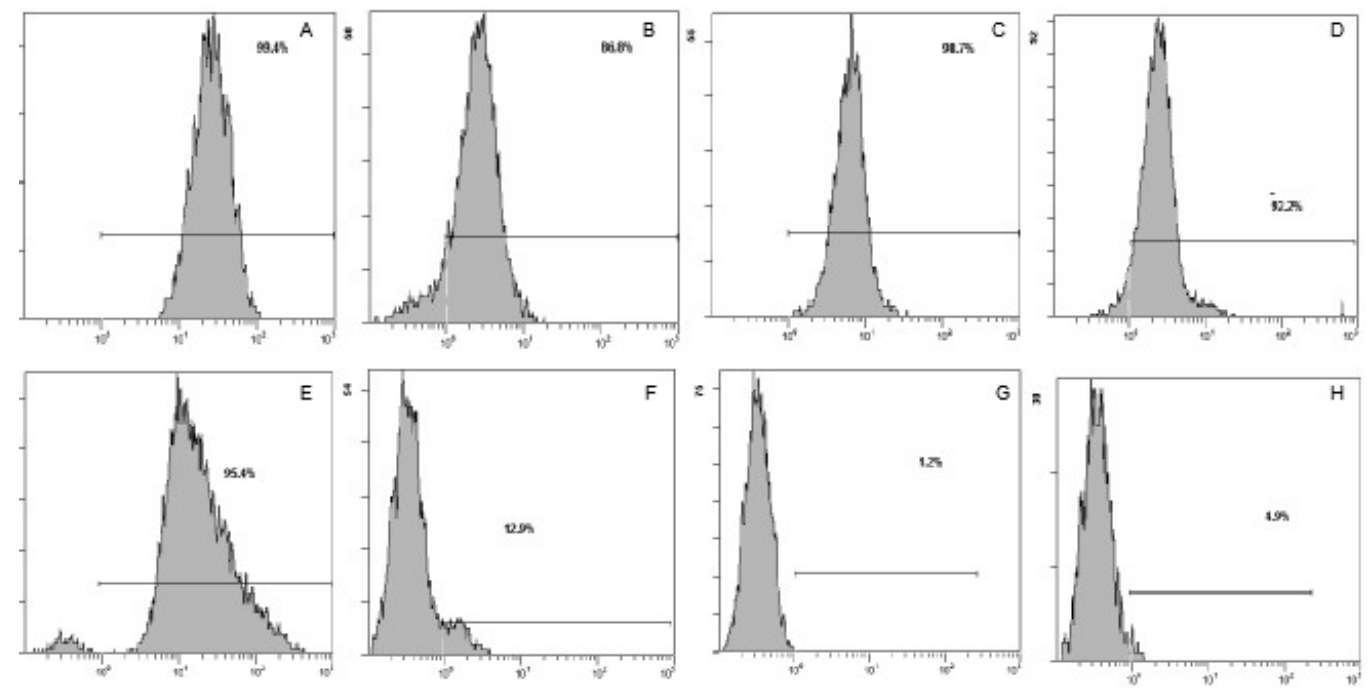

Figure 2. Immunophenotype validation of UC-MSCs.

\section{Validation of EPO gene transfection in human UC-MSCs}

Expression of the EPO protein could be detected in pcDNA3-EPO plasmid-transfected UC-MSCs using western blotting (Figure 3). The position of the protein band corresponding to 34 kDa correlated with the EPO bands from human fetal liver cell L02 (positive control) and no EPO protein was detected in untransfected human UC-MSCs (negative control).

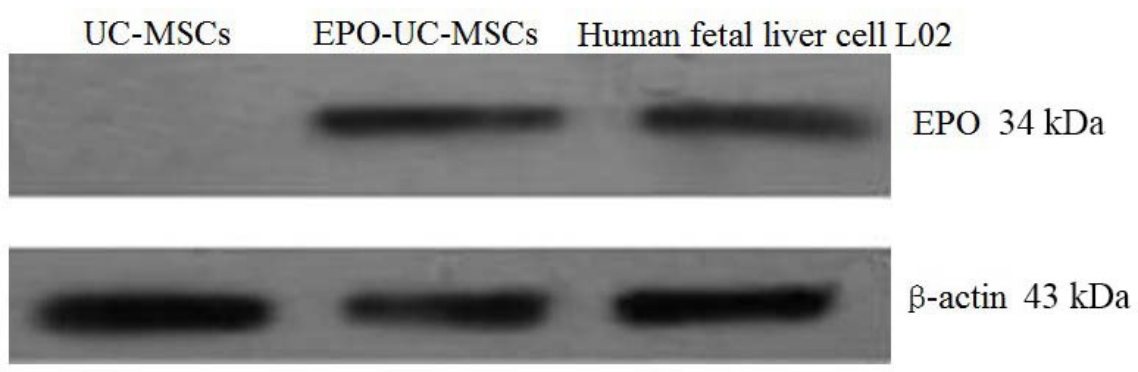

Figure 3. Expression of the EPO protein in EPO-transfected UC-MSCs. 


\section{Angiography of rats' artery}

Results of angiography of femoral artery of rat subjects are shown in Figure 4. In ischemia control group, femoral artery and its branches could not be found. In EPO- and UC-MSC-treated groups, moderate neovascularization could be observed at similar levels. In the EPO-transfectedUC-MSC-treated group, there was large amount of neovascularization with abundant collateral circulation.

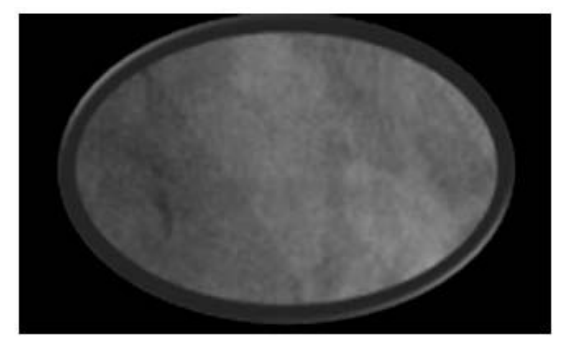

Ischemia control group

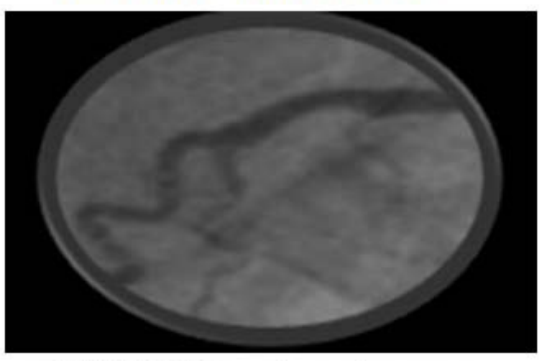

UC-MSC treatment group

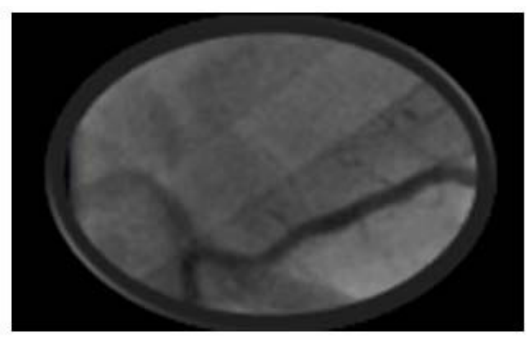

EPO treatment group

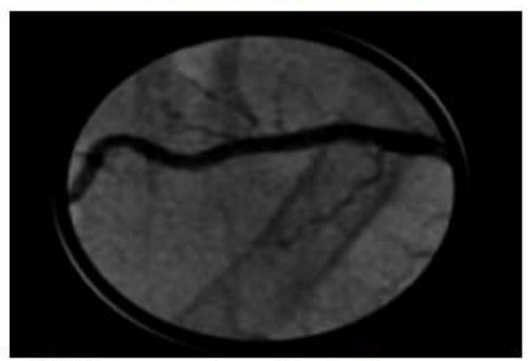

EPO gene transfected

UC-MSC treatment group

Figure 4. Angiography of femoral artery of rats.

\section{Transplantation of EPO-transfected-UC-MSC causes neovascularization in ischemic limbs}

Cells positive for CM-Dil and neovascularization were counted using a fluorescence microscope at high magnification (400X). The mean count in the gastrocnemius tissue sections was $(0+0) / \mathrm{HP}$ (high power field) in the ischemia control, $(0+0) / \mathrm{HP}$ in the EPO-treated group, $(32.46$ $+6.68) / H P$ in the UC-MSC-treated group, and $(59.64+10.38) / H P$ in the EPO-transfected-UCMSC-treated group. The difference was statistically significant $(P<0.05)$. Representative images are shown in Figure 5.

The neovascularization count was $(9.3+0.6) / \mathrm{HP}$ in the ischemia control, $(20.1+$ 1.4)/HP in the EPO-treated group, $(19.9+2.4) / \mathrm{HP}$ in the UC-MSC-treated group, and (40.2 $+3.8 / \mathrm{HP}$ in the EPO-transfected-UC-MSC-treated group. Compared with the control group, neovascularization in the EPO- and UC-MSCs-treated groups had increased. However, the effect of these two treatments was similar $(P>0.05)$. The EPO-transfected-UC-MSC-treated 
group can further enhance the curative effect of treatment group $(P<0.05)$. Representative images are presented in Figure 6.

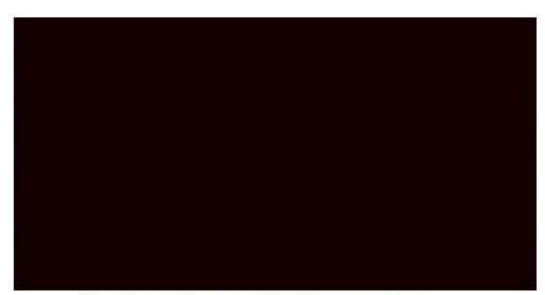

Ischemia control group

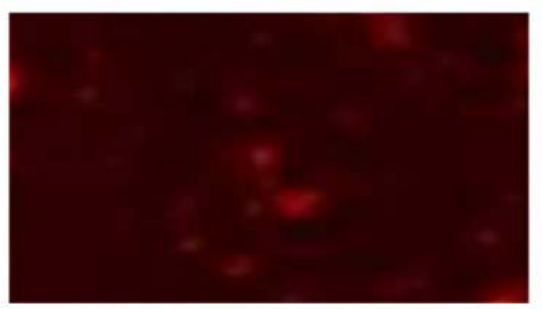

UC-MSC treatment group

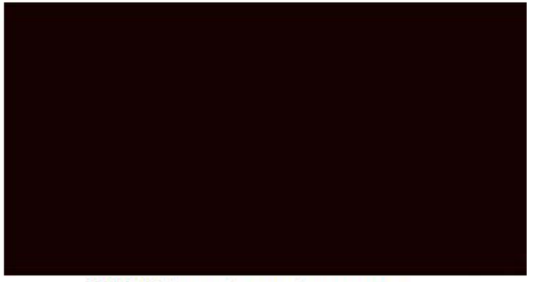

EPO treatment group

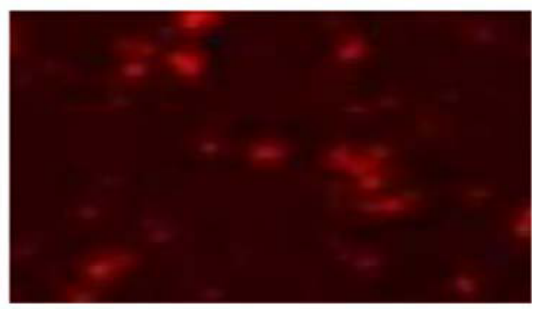

EPO gene transfected

UC-MSC treatment group

Figure 5. CM-Dil-positive cells in the gastrocnemius muscle of rats at 4 weeks post-surgery (immunofluorescence, 400X).

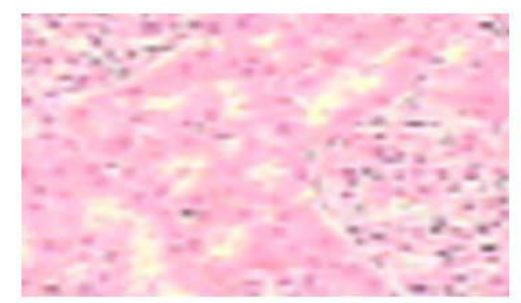

Ischemia control group

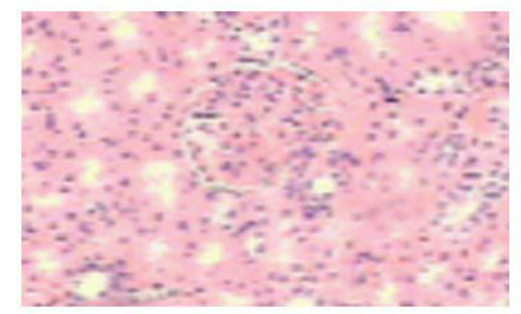

UC-MSC treatment group

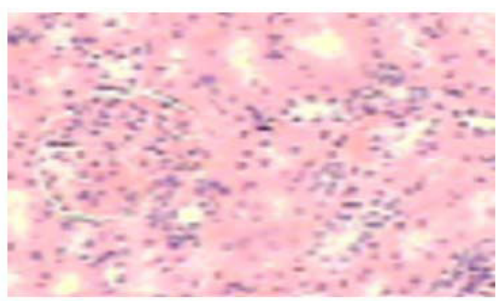

EPO treatment group

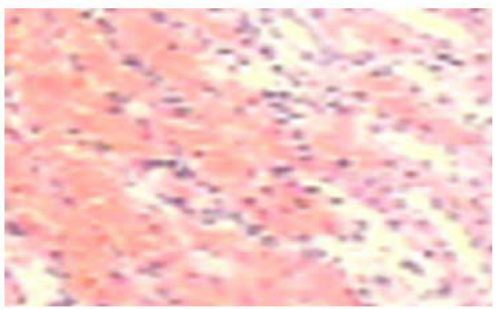

EPO gene transfected

UC-MSC treatment group

Figure 6. Quantification of neovascularization in rats at 4 weeks post-surgery (immunohistochemical staining, 400X). 


\section{Detection of in vivo EPO gene expression in transplanted rats}

cDNA-specific primers were used for EPO gene amplification in ischemia-treated tissues (Figure 7). RT-PCR revealed higher EPO expression in EPO-transfected-UC-MSCs-treated group than EPO-treated group. The relative content of EPO mRNA was $0.79+0.06$ and $0.19+0.04$, respectively $(P<0.05)$.

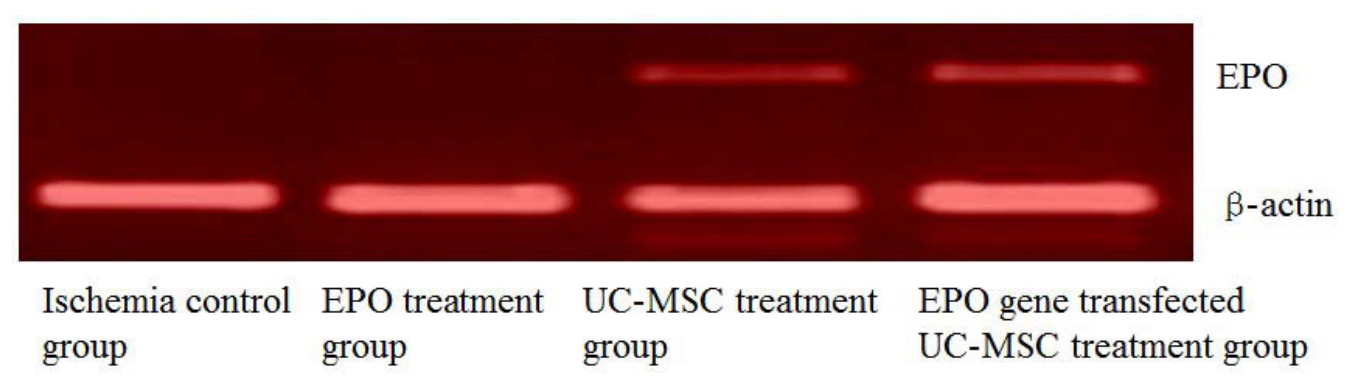

Figure 7. EPO gene expression analysis in ischemia treated rat tissues.

\section{DISCUSSION}

Limb ischemia is the most common vascular disease that requires vascular surgery, and it is caused by the stenosis of limb arteries or aorta, occlusion, or spasm-caused insufficient blood supply to the limb (Sun et al., 2006a; Zhang et al., 2007; Liu et al., 2014). Currently drug therapy, surgery, interventional therapy, gene therapy and stem cell therapy have been used clinically. While it can be divided into acute and chronic limb ischemias according to the onset timeframe, according to the lesion position, it can be divided into upper and lower limb ischemias. Lower limb ischemic disease tends to have spread in recent years, in which case, most patients face threat of amputation at final stages and their quality of life gets greatly affected. Treatment methods for this disease need improvement. In recent years, a large number of studies have shown that stem cells can promote angiogenesis under certain conditions, thus paving a new way for the treatment of limb ischemia (Sun et al., 2006b; Zhu et al., 2009; Chang et al., 2013). Umbilical cord mesenchymal stem cells have strong ability to proliferate and differentiate in vitro and have displayed good efficacy in the treatment of limb ischemia (Ha et al., 2001; Chen et al., 2009; Kim et al., 2010).

Erythropoietin (EPO) is a relatively new neurotransmitter, which acts by binding with specific erythropoietin receptors on the cell surface (Buzahska et al., 2006; Kristal et al., 2008). EPO and its receptors are widely distributed in human and animal brains with autocrine or paracrine roles in human and rat limb ischemia and hypoxia. While endogenous EPO-R has a protective role, exogenous EPO can enhance this effect. Studies have reported that under ischemic conditions, EPO can inhibit free radical chain reactions, improve intracellular calcium ion overload, reduce damage to ischemic area induced by injury endogenous factors, and inhibit ischemia- and hypoxiainduced apoptosis (Kaptanoglu et al., 2004; Gorio et al., 2005).

Establishing an animal model of limb ischemia is an effective way to investigate ischemic diseases. In this study, transplantation of EPO-transfected UC-MSCs was used for treating limb ischemia in rat models. Human umbilical cord mesenchymal stem cells and EPO were chosen based on their good effect of stem cell transplantation therapy as mentioned above. The EPO 
gene-transfected human umbilical cord mesenchymal stem cells could stably express EPO in vitro. At 4 weeks after transplantation, angiography showed moderate amount of newborn vessels EPO gene- and UC-MSC-treated groups, with similar efficacy. Interestingly, a large number of newborn vessels and rich collateral circulation could be visualized in the EPO-transfected-UC-MSC-treated group. Measurement of capillary density in transplanted rat tissues confirmed the angiography results. RT-PCR detected relatively higher EPO gene expression in vivo in EPO-transfected-UCMSC-treated group than the EPO-treated group. Taken together, the results demonstrate that EPO gene injection or UC-MSCs treatment can promote angiogenesis but can have an enhanced effect when used in combination, i.e., EPO-transfected-UC-MSCs transplantation. This seems to have a protective effect on ischemic tissues, thus providing a new experimental basis for treating limb ischemia.

\section{Conflicts of interest}

The authors declare no conflict of interest.

\section{REFERENCES}

Bieback K, Kern S, Klüter H and Eichler H (2004). Critical parameters for the isolation of mesenchymal stem cells from umbilical cord blood. Stem Cells 22: 625-634.

Buzańska L, Jurga M and Domańska-Janik K (2006). Neumnal differentiation of human umbilical cord blood neural stem -like cell line. Neurodegener. Dis. 3: 19-26.

Chang XP, Ma Y, Bi XJ, Song LJ, et al. (2013). Human umbilical cord mesenchymal stem cells: Comparison of hematopoiesis supporting capacity before and after cryopreservation. Chin. J. Tissue Eng. Res. 32: 5765-5771.

Chen L, Qv ZN, Zhang DQ, Lu H, et al. (2005). Culture and identification of mesenchymal stem cells derived from human umbilical blood. Jiangsu Med. J. 31: 481-483.

Chen L, Hui GZ, Zhang S, Miao ZN, et al. (2009). HUCB-MSCs transplants promote neurological functional recovery after traumatic brain injury. Chin. J. Traumatol. 25: 498-502.

Chi ZH, Zhang H, He DM and Lu Y (2006). Optimal culture of human umbilical cord blood mesenchymal stem cells. Chin. J. Pract. Intern. Med. 26: 372-375.

Cui B, Li E, Yang B and Wang B (2014). Human umbilical cord blood-derived mesenchymal stem cell transplantation for the treatment of spinal cord injury. Exp. Ther. Med. 7: 1233-1236.

Gorio A, Madaschi L, Di Stefano B, Carelli S, et al. (2005). Methylprednisolone neutralizes the beneficial effects of erythropoietin in experimental spinal cord injury. Proc. Natl. Acad. Sci. U. S. A. 102: 16379-16384.

Ha Y, Choi JU, Yoon DH, Yeon DS, et al. (2001). Neural phenotype expression of cultured human cord blood cells in vitro. NeuroReport 12: 3523-3527.

Jin W, Yang AH and Xing YQ (2007). Culture and identification of mesenchymal stem cells derived from umbilical cord blood. Med. J. Wuhan Univ. 28: 488-491.

Jin W, Kong J, Lu T, Wang H, et al. (2011). Erythropoietin prevents secondary brain injury induced by cortical lesion in mice: possible involvement of Nrf2 signaling pathway. Ann. Clin. Lab. Sci. 41: 25-32.

Kaptanoglu E, Solaroglu I, Okutan O, Surucu HS, et al. (2004). Erythropoietin exerts neuroprotection after acute spinal cord injury in rats: effect on lipid peroxidation and early ultrastructural findings. Neurosurg. Rev. 27: 113-120.

Kidd SA (1995). Congenital heart disease: a 10 year cohort. J. Paediatr. Child Health 31: 362.

Kim JY, Jeon HB, Yang YS, Oh W, et a1. (2010). Application of human umbilical cord blood-derived mesenchymal stem cells in disease models. World J. Stem Cells 2: 34-38.

Kristal B, Shurtz-Swirski R, Tanhilevski O, Shapiro G, et al. (2008). Epoetin-alpha: preserving kidney function via attenuation of polymorphonuclear leukocyte priming. Isr. Med. Assoc. J. 10: 266-272.

Li JB, Jiao HL, Shan H and Zhao LN (2010). Umbilical cord blood mesenchymal stem cells on the distribution of the main organs of mice model of cerebral apoplexy. Chin. J. Blood Transfus. 23: 870-871.

Liu K, Luo J, Wang Y, Li XX, et al. (2014). Allogeneic bone marrow stromal cells transplantation for the treatment of hind limb ischemia in rats. China J. Vasc. Surg. 6: 88-92. 
Marfia G, Madaschi L, Marra F, Menarini M, et al. (2011). Adult neural precursors isolated from post mortem brain yield mostly neurons: An erythropoietin-dependent process. Neurobiol. Dis. 43: 86-98.

Park H, Temenoff JS, Tabata Y, Caplan Al, et al. (2007). Injectable biodegradable hydrogel composites for rabbit marrow mesenchymal stem cell and growth factor delivery for cartilage tissue engineering. Biomaterials 28: 3217-3227.

Park Y, Chen Y, Ordovas L and Verfaillie CM (2014). Hepatic differentiation of human embryonic stem cells on microcarriers. J. Biotechnol. 174: 39-48.

Sun HM, Ji FQ, Li RP, Wang Q, et al. (2006a). Difference of human umbilical cord blood derived mesenchymal stem cells in differential culture conditions. China Clin. Rehab. 10: 51-53.

Sun ZF, Jiang W, Wang HT and Han DE (2006b). SDF-1 combined with bone marrow mononuclear cell transplantation for the treatment of hindlimb ischemia in rats. Chin. J. Gen. Surg. 21: 526-529.

Zhang YH, Dai KY and Mei JC (2007). Vascular endothelial growth factor combined with bone marrow mononuclear cell autograft for limb ischemia in rats. Chin. J. Tissue Eng. Res. 15: 2882-2885.

Zhu YH, Feng SQ, Sun XH, Wang X, et al. (2009). Repair of spinal cord injury with co-transplantation of human umbilical cord mesenchymal stem cells and autologously activated Schwann cells. Chin. J. Orthop. Trauma 11: 747-751. 